\title{
NGHIÊN CỨU MỐI QUAN HẸ BÁO CÁO BỀN VỮNG VÀ HIỆU QUẢ TÀI CHÍNH TIẾP CẬN THEO PHƯƠNG PHÁP META-ANALYSIS
}

\author{
RESEARCH ON SUSTAINABLE REPORTING RELATIONSHIP AND FINANCIAL PERFORMANCE \\ APPROACH META-ANALYSIS METHODOLOGY
}

Nguyễn Văn Linh, Đặng Ngọc Hùng*, Đặng Thị Hậu

\section{Tóm TẮT}

Mối quan hệ giữa báo cáo bền vững (SR) và hiệu quả tài chính doanh nghiệp (CFP) từ lâu đã trở thành một cuộc tranh luận trọng tâm và gây tranh cãi trong các nghiên cứu. Tuy nhiên, các nghiên cứu thực nghiệm trước đây đưa ra kết luận không đồng nhất. Mục đích của nghiên cứu này là xem xét một cách có hệ thống và định lượng mối liên kết SR-CFP trong một khung phân tích tổng hợp. Dựa trên 30 nghiên cứu, nghiên cứu này ước tính rằng quy mô ảnh hưởng tổng thể của mối quan hệ SR-CFP là tích cực và có ý nghĩa, do đó chứng thực lập luận rằng SR đã nâng cao hiệu quả tài chính. Hơn nữa, nghiên cứu này làm sáng tỏ mối quan hệ nhân quả giữa SR và CFP, phát hiện này ủng hộ lý thuyết các bên liên quan.

Từ khóa: Báo cáo bền vững; hiệu quả tài chính; trách nghiệm xã hội; phân tích tổng hợp.

\section{ABSTRACT}

The relationship between sustainability reporting (SR) and corporate financial performance (CFP) has long been a central and controversial debate in studies. However, previous experimental studies have reached mixed conclusions. The purpose of this study is to systematically and quantitatively examine the SR-CFP link in a meta-analytical framework. Based on 30 studies, this study estimates that the overall impact size of the SR-CFP relationship is positive and significant, thus corroborating the argument that the SR has enhanced financial performance. Furthermore, this work sheds light on the causal relationship between SR and CFP, a finding that supports the stakeholder theory.

Keywords: Sustainability report; financial performance; social responsibility; meta-analysis.

Trường Đại học Công nghiệp Hà Nội

*Email: hungdangngockt@yahoo.com.vn; dangngochung@haui.edu.vn

Ngày nhận bài: 18/01/2021

Ngày nhận bài sửa sau phản biện: 23/6/2021

Ngày chấp nhận đăng: 25/8/2021

\section{GIỚI THIÊU}

Thị trường ngày càng trở nên cạnh tranh và tốc độ thay đổi nhanh chóng đang đặt các công ty dưới áp lực chưa từng có để không chỉ thành công mà còn duy trì thành công của họ trong tương lai. Báo cáo bền vững (SR) đã được chú ý nhiều trong những năm gần đây, khi các công ty, nhà đầu tư và người tiêu dùng đều hướng sự chú ý của họ đến tính bền vững của doanh nghiệp ngày càng quan trọng [2, 35]. Các công ty được kỳ vọng sẽ vượt ra khỏi trọng tâm tài chính hẹp và ngắn hạn, mà vươn ra sự bền vững về kinh tế, môi trường và xã hội [23]. Phát triển các chiến lược công ty để làm tốt hơn và biến các công ty thành các tổ chức có trách nhiệm quan tâm đến môi trường, và khía cạnh xã hội ngày càng trở thành một điều bắt buộc hơn là một lựa chọn để dẫn đầu trong các thị trường trong tương lai $[9,11]$. Tính bền vững được định nghĩa là đáp ứng nhu cầu của chúng ta ngày nay mà không ảnh hưởng đến khả năng đáp ứng của các thế hệ tương lai [24]. Tính bền vững của doanh nghiệp là mở rộng điểm mấu chốt về tài chính thành điểm mấu chốt bao gồm các khía cạnh môi trường và xã hội của hiệu quả hoạt động của doanh nghiệp [1]. Khi các công ty cố gắng duy trì sự phù hợp trong các thị trường đang thay đổi, họ nhận ra rằng không còn đủ để chỉ tập trung vào tính kinh tế của doanh nghiệp [18]. Xây dựng một doanh nghiệp vững mạnh trên cơ sở chiến lược ngày càng trở nên phụ thuộc vào việc một công ty định vị bản thân tốt như thế nào về phát triển bền vững cân bằng giữa tài chính, môi trường và phát triển con người [43].

Tại sao một phân tích tổng hợp chúng tôi thực hiện là là cần thiết? Trong khi $[36,40,49]$ đã xem xét hiệu quả điều tiết của các biện pháp $C S R$, nghiên cứu này mở rộng các nghiên cứu trên bằng cách xem xét các thước đo khác nhau về hiệu quả tài chính, xem xét cách tiếp cận đo lường SR theo khía cạnh tổng thể và theo từng thành phần, đồng thời xem xét mối quan hệ giữa SR-CFP và CFP -SR. Ngoài ra, để giải quyết mối quan tâm của [46], các đặc điểm của mẫu (ví dụ, cỡ mẫu, cách thức đo lường SR và CFP và quốc gia). Bên cạnh đó nghiên cứu đã cập nhật những nghiên cứu được xuất bản mới nhất, báo cáo về tính bền vững đã tăng lên đáng kể trong những năm gần đây, đặc biệt là tại các nước đang phát triển.

Nghiên cứu này đóng góp những tri thức mới và thông qua những phát hiện thú vị về mối quan hệ SR-CFP trên cơ 
sở phân tích tổng hợp cung cấp một đánh giá chặt chẽ hơn về nghiên cứu trong quá khứ. Các bài báo tổng quan tài liệu truyền thống về mối quan hệ SR-CFP chủ yếu dựa vào các bài phê bình tường thuật. Tóm tắt từ các bài phê bình tài liệu tự sự không xem xét các lỗi lấy mẫu và đo lường và các suy luận sai lầm có thể được rút ra từ các bài phê bình đó. Tuy nhiên, phân tích tổng hợp xem xét sự thay đổi của nghiên cứu chéo trong mối tương quan giữa $S R$ và CFP và do đó đưa ra các kết luận đáng tin cậy hơn.

\section{CƠ SỞ LÝ THUYẾT}

Ullmann [46] cho thấy sự cần thiết phải có một lý thuyết về hiệu quả hoạt động xã hội của doanh nghiệp vì những phát hiện không nhất quán là kết quả của các nghiên cứu về mối quan hệ lẫn nhau giữa sự công bố thông tin về hoạt động xã hội, hiệu quả hoạt động xã hội và hiệu quả kinh tế của các công ty Hoa Kỳ. Trong số các lý thuyết đã được sử dụng trong các tài liệu trước đây, có ba lý thuyết nhận được nhiều sự quan tâm của các nhà đầu tư, đó là lý thuyết đại diện, lý thuyết tính hợp pháp và lý thuyết các bên liên quan.

Lý thuyết đại diện do Jensen \& Meckling phát biểu rằng một trong những chức năng chính của nhà quản lý là gắn lợi ích của công ty với lợi ích của cổ đông [32]. Friedman đã sử dụng lý thuyết đại diện để xem xét hoạt động của các công ty trong trách nhiệm xã hội của doanh nghiệp (CSR). Friedman khẳng định rằng tham gia vào CSR là dấu hiệu của một vấn đề của đại diện hoặc xung đột giữa lợi ích của nhà quản lý và cổ đông [21]. Ông cho rằng các nhà quản lý sử dụng CSR như một phương tiện để theo đuổi các mục tiêu xã hội, kinh tế, chính trị và nghề nghiệp của họ. Theo quan điểm này, nguồn lực đầu tư vào CSR sẽ được sử dụng một cách khôn ngoan hơn, từ góc độ xã hội, vào việc cải thiện hiệu quả của công ty. Ông lập luận thêm rằng những đồng tiền được sử dụng trong các hoạt động CSR chỉ là tiêu tiền của người khác và không mang lại nhiều lợi ích cho công ty nói chung. Lý thuyết này cho rằng, các chi phí môi trường được sử dụng để bảo vệ/cải thiện môi trường như giảm thiểu ô nhiễm hoặc giảm phát thải không khí được dự đoán sẽ làm tăng chi phí sản xuất và do đó làm giảm hiệu quả kinh tế. Lý thuyết đại diện đã được thử nghiệm trong một số nghiên cứu xem xét mối quan hệ SR và CFP. Ví dụ, Wright \& Ferris nhận thấy rằng giá cổ phiếu phản ứng tiêu cực với các thông báo về việc thoái vốn của các đơn vị kinh doanh ở Nam Phi, điều mà ho hiểu là phù hợp với lý thuyết đại diện [51]. Các nghiên cứu của [31,34] cũng tìm thấy mối quan hệ tiêu cực giữa hoạt động môi trường và hoạt động kinh tế.

Lý thuyết hợp pháp, do Davis khởi xướng, phát biểu rằng "xã hội trao quyền hợp pháp và quyền lực cho doanh nghiệp. Về lâu dài, những người không sử dụng quyền lực theo cách mà xã hội coi là có trách nhiệm sẽ có xu hướng mất quyền lực" [16]. Lý thuyết hợp pháp cho rằng các tổ chức liên tục tìm cách đảm bảo rằng họ hoạt động trong giới hạn và chuẩn mực của xã hội tương ứng [17]. Theo lý thuyết hợp pháp, các tập đoàn phải có trách nhiệm với xã hội và chịu trách nhiệm trước xã hội để hoạt động kinh doanh hợp pháp $[14,44]$.
Lý thuyết các bên liên quan, lần đầu tiên được đề xuất bởi Freeman, đưa ra một cuộc thảo luận về mối liên hệ giữa các bên liên quan bên ngoài và các chức năng của công ty. Freeman định nghĩa các bên liên quan là "bất kỳ nhóm hoặc cá nhân nào có thể ảnh hưởng hoặc bị ảnh hưởng bởi việc đạt được các mục tiêu của tổ chức", các nhà hoạch định chính sách và cổ đông, phương pháp tiếp cận các bên liên quan được đề xuất là lý thuyết tốt nhất để giải thích hành vi của nhà quản lý [20].

\section{TỔNG QUAN VÀ GIÁ THUYẾT NGHIÊN CỨU}

Trong phần này, các tác giả sẽ tổng quan ngắn gọn về mối liên hệ giữa $S R$ và $C F P$, các kết quả thực nghiệm thường hỗn hợp. Nhiều nghiên cứu xem xét mối quan hệ SR và CFP đã sử dụng SR như là biến phụ thuộc và các tham số CFP là biến độc lập [37, 39]. Trong khi một số nghiên cứu khác sử dụng SR là biến độc lập và CFP là biến phụ thuộc $[3,4]$. Kết quả của các nghiên cứu đề cập ở trên là mang tính hỗn hợp. Cụ thể, một vài nghiên cứu tìm thấy mối tương quan dương giữa hai biến $[40,48]$. Mặt khác, một luồng nghiên cứu bền vững khác đã tập trung vào mối quan hệ giữa kết quả hoạt động môi trường và CFP. Một nhóm các nhà nghiên cứu đã ghi nhận rằng hoạt động môi trường của các công ty làm tăng CFP của các công ty. Một nhóm các nhà nghiên cứu khác đã ghi nhận rằng hoạt động tài chính có liên quan tiêu cực đến hoạt động môi trường chẳng hạn như chỉ số ô nhiễm. Tóm lại, từ các kết quả khác nhau của các nghiên cứu trên và để hiểu rõ hơn bản chất mối quan hệ SR và CFP, nghiên cứu này sẽ xem xét mối quan hệ SR và CFP theo những nội dung sau:

\subsection{Nghiên cứu về ảnh hưởng báo cáo bền vững tới và hiệu quả tài chính}

Có rất nhiều nghiên cứu trước đây với kết quả mẫu thuẫn nhau khi đưa ra các mối quan hệ có tương quan âm, dương hay thậm chí là không có mối quan hệ nào giữa trách nhiệm xã hội và hiệu quả hoạt động như các nghiên cứu của $[6,39,41,48]$. Như vậy, có thể chia thành ba nhóm nghiên cứu như sau:

- Nhóm thứ nhất: nghiên cứu cho rằng mối quan hệ SR và hiệu quả hoạt động thu được kết quả ngược chiều [2]. Rhou, Singal \& Koh muốn nhấn mạnh vào hoạt động chiến lược của doanh nghiệp cũng như việc điều hành cần dựa vào các nguồn lực của mình để gia tăng lợi nhuận cho cổ đông và nhà đầu tư [42]. Bên cạnh đó các nghiên cứu nhấn mạnh về việc sử dụng và tìm cách phân phối tối ưu nguồn lực khan hiếm sẽ có ảnh hưởng tiêu cực đến CFP. Tầm quan trọng của công tác truyền thông trong các hoạt động SR cần được xem xét và chú trọng đối với các bên liên quan.

- Nhóm thứ hai: Dựa vào lý thuyết các bên liên quan của Freeman cho rằng có mối quan hệ thuận chiều tích cực giữa SR và CFP gồm các nghiên cứu $[6,7,13,19,28,37,41$, $48,50]$. Theo lợi ích của cổ đông, khi công ty quyết định thực hiện các hoạt động xã hội thì cần quan tâm đến các đối tượng và các bên liên quan khác như khách hàng, nhân viên, nhà cung cấp, cộng đồng... Hoạt động SR sẽ góp 
phần giúp công ty gia tăng CFP bằng việc tăng doanh thu tăng hình ảnh công ty, thương hiệu và danh tiếng...

- Nhóm thứ ba: không có cùng quan điểm nghiên cứu với hai nhóm trên, nhóm nghiên cứu thứ ba cho rằng, không có mối quan hệ cụ thể nào giữa SR và CFP như [45]. Ở những nghiên cứu này, lý do không tìm thấy mối liên hệ giữa $S R$ và $C F P$ là vì có quá nhiều yếu tố ảnh hưởng lên hiệu quả hoạt động $[5,8,10]$.

\subsection{Nghiên cứu về ảnh hưởng của hiệu quả tài chính đến báo cáo bền vững}

Đối với các doanh nghiệp theo đuổi động cơ lợi nhuận doanh nghiêp đầu tư vào hoạt động phát triển bền vững sẽ thay đổi kết quả tài chính trong tương lai của doanh nghiệp theo chiều hướng tích cực. Ví dụ, đầu tư vào phát triển bền vững sẽ gia tăng hình ảnh của doanh nghiệp với cộng đồng từ đó tăng doanh thu, thị phần hoặc sẽ thu hút nhân viên giỏi làm việc tại đơn vị, hoặc giảm những xung đột không mong muốn với các bên liên quan, hoặc trách được những rắc rối về mặt pháp lý. Chính ví vậy giá trị của doanh nghiệp sẽ có quan hệ với mức độ mà doanh nghiệp đầu tư vào hoạt động phát triển bền vững. Có nhiều nghiên cứu đã tìm hiểu mối quan hệ giữa CFP với kết quả thực hiện SR như $[12,29]$, hay mối quan hệ giữa CFP với SR của $[22,25]$. Theo $[4,47]$ cho rằng, CFP gia tăng sẽ làm tăng việc công bố thông tin về môi trường, xã hội và quản trị của công ty, đồng thời kết quả nghiên cứu cho thấy CFP có ảnh hưởng thuận chiều với mức độ công bố thông tin trong báo cáo bền vững. Morhardt đã thực hiện nghiên cứu tổng quan trên cơ sở xem xét 101 bài báo có liên quan đến SR và CFP trong giai đoạn 1992 -2011, cho thấy trong giai đoạn đầu nghiên cứu chủ yếu tập trung ở những phát triển, tuy nhiên từ ở giai đoạn từ năm 2000 trở lại đây cũng đã bắt đầu có những nghiên cứu tại nước đang phát triển, và cần có thêm những nghiên cứu bổ sung về chủ đề này [38].

\subsection{Nghiên cứu về mối quan hệ báo cáo bền vững và hiệu quả tài chính}

Nghiên cứu mối quan hệ hai chiều giữa $S R$ và $C F P$, đã có một vài nghiên cứu tìm thấy mối tương quan dương giữa hai biến $[40,48]$, nhưng một số nghiên cứu lại không tìm thấy mối quan hệ nào giữa hai biến [37], nhưng cũng có những nghiên cứu tìm thấy mối tương quan âm giữa hai biến $[15,33]$. Trên cơ sở các lý thuyết, tổng quan nghiên cứu với những tranh luận và các kết quả thực nghiện về mối quan hệ giữa SR và CFP, chúng tôi xây dựng giả thuyết nghiên cứu như sau:

Giả thuyết $H_{1}$ : Có mối quan hệ thuận chiều giữa hiệu SR với CFP.

Giả thuyết $\mathrm{H}_{2}$ : Có mối quan hệ thuận chiều giưa hiệu CFP với $S R$.

\section{PHƯƠNG PHÁP NGHIÊN CỨU}

\subsection{Dữ liệu nghiên cứu}

Chúng tôi đã xác định các nghiên cứu thực nghiệm còn tồn tại tập trung vào mối quan hệ giữa báo cáo bền vững và hiệu quả tài chính doanh nghiệp. Để thu thập càng nhiều bài báo có liên quan càng tốt từ các tài liệu hiện có, chúng tôi đã tiến hành tìm kiếm từ khóa của cơ sở dữ liệu điện tử thích hợp cho cả bài báo đã xuất bản và chưa xuất bản (đang hoạt động). Để xác định các bài báo đã xuất bản cho dữ liệu phân tích tổng hợp, vì nghiên cứu này chỉ tập trung vào một mối quan hệ báo cáo bền vững của công ty và hiệu quả tài chính của công ty nên chúng tôi đã sử dụng các từ hoặc thuật ngữ sau để tìm kiếm các bài báo đã xuất bản trong cơ sở dữ liệu Google Scholar, ProQuest, EBSCO, Science Direct, Emerald, JSTOR, Springer, Scopus bao gồm các cụm từ "báo cáo bền vững", "báo cáo phát triển bền vững", "trách nhiệm xã hội", "hiệu quả kinh doanh", "hiệu quả tài chính" và "giá trị doanh nghiệp". Sau khi tìm kiếm và thu thập các bài báo có liên quan trong nghiên cứu này, chúng tôi đã đánh giá mức độ phù hợp của từng nghiên cứu với trọng tâm nghiên cứu của chúng tôi để xác định xem đó có phải là một nghiên cứu đủ điều kiện để được đưa vào phân tích tổng hợp hay không. Để đủ điều kiện, một nghiên cứu phải đáp ứng ba tiêu chí: (1) nghiên cứu phải có kết quả cụ thể mối quan hệ SR-CFP, vì bài báo của chúng tôi chỉ tập trung vào mối quan hệ SR-CFP; (2) nghiên cứu phải có sẵn trên mạng ở dạng toàn văn; và (3) nghiên cứu kiểm tra mối quan hệ SR-CFP phải có mối tương quan (r) hoặc số liệu thống kê tương đương được báo cáo. Các giá trị tương đương $r$ này có thể là giá trị t-student $(\mathrm{t})$, giá trị p, hệ số beta hoặc Chi-bình phương (v2) vì giá trị hệ số tương quan (r) là cần thiết khi tiến hành các quy trình phân tích tổng hợp. Các nghiên cứu được chọn để đưa vào phân tích tổng hợp trên cơ sở ba tiêu chí đã được đề cập ở trên. Đầu tiên, phân tích tổng hợp chỉ bao gồm những nghiên cứu thực nghiệm báo cáo cỡ mẫu và thống kê kết quả $(r, F$ đơn biến, $\mathrm{t}, \mathrm{X}^{2}$ ) cho phép tính toán hệ số tương quan với các công thức được cung cấp bởi Hunter \& Schmidt [30]. Sau khi hoàn thành các thủ tục truy xuất dữ liệu, các tác giả đã thu được 30 nghiên cứu. Bộ dữ liệu các nghiên cứu được tóm tắt trong phụ lục 1 . Để giảm lỗi mã hóa, các tác giả đã chuẩn bị một giao thức mã hóa xác định thông tin được trích xuất từ mỗi nghiên cứu. Mẫu mã đã được chuẩn bị hoặc những người viết mã đã ghi lại dữ liệu trích xuất về các biến quan tâm, bao gồm kích thước ảnh hưởng, kích thước mẫu và đặc điểm nghiên cứu.

\subsection{Phân tích dữ liệu}

Các tác giả đã tiến hành phân tích tổng hợp này theo hướng dẫn của Hunter \& Schmidt [30]. Trước hết, chúng tôi chuyển đổi số liệu thống kê được báo cáo thành một kích thước hiệu ứng chung. Để giải thích độ lệch của phân phối các hệ số tương quan mẫu, chúng tôi chuyển đổi mối tương quan thành hệ số Fisher's $z$ với công thức $Y_{i}=0,5 \log \left(1+r_{i} / 1-r_{i}\right)$. Sau đó, các tác giả tính trung bình và trọng số các hệ số $z$ theo công thức $V_{i}=1 / N-3$. Có hai mô hình được sử dụng là mô hình hiệu ứng cố định và mô hình tác động ngẫu nhiên. Phân tích tổng hợp các hiệu ứng cố định giả định rằng các kích thước ảnh hưởng của quần thể là bằng nhau cho tất cả các nghiên cứu, vì vậy sự khác biệt về các kích thước ảnh hưởng quan sát được là do lỗi lấy mẫu. Đối với mô hình tác động ngẫu nhiên, kích thước ảnh 
hưởng của quần thể có thể khác nhau giữa các nghiên cứu [26]. Sự khác biệt về kích thước hiệu ứng quan sát được là do sự kết hợp của sự khác biệt thực sự (thành phần phương sai) và sai số lấy mẫu. Nó thường được ưu tiên về mặt phương pháp luận, các tác giả đã tiến hành kiểm tra tính đồng nhất và để xác định xem nên sử dụng mô hình hiệu ứng cố định hay ngẫu nhiên. Tuy nhiên, trong nghiên cứu này chúng tôi trình bày cả hai mô hình hiệu ứng cố định và hiệu ứng ngẫu nhiên, các kết quả để nhận xét, đánh giá dựa trên mô hình hiệu ứng ngẫu nhiên.

\section{KẾT QUẢ NGHIÊN CỨU VÀ THẢO LUÂN}

Dựa trên cơ sở dữ liệu của 30 mẫu nghiên cứu, chúng tôi thực hiện bổ sung các phân tích về mối quan hệ giữa SR và $C F P$, kết quả được trình bày ở phụ lục 2,3 trên quy mô mẫu tổng thể, cho thấy SR và CFP có quan hệ thuận chiều, với $r=0,29$, với khoảng tin cậy $95 \%$ nằm trong khoảng $[0,21-0,36]$ với mẫu t. Với hệ số $I^{2}=87,53 \%$ theo Higgins \& Thompson [27] sự hiện diện của tính không đồng nhất ở mức cao. Bên cạnh đó, chúng tôi chia thành 2 nhóm, nhóm 0 có 4 nghiên cứu ảnh hưởng của CFP đến SR và nhóm 1 có 26 nghiên cứu xem xét ảnh hưởng của SR đến CFP, kết quả phụ lục 3 cho thấy đều có mối quan hệ tích cực giữa SR và CFP, trong đó mối quan hệ SR đến CFP có tương quan mạnh hơn.

Để đánh giá khả năng thiên vị trong xuất bản (publication bias), chúng ta biết rằng khi một kết quả nghiên cứu cho kết quả "negative" thì công trình nghiên cứu đó khó có cơ hội được công bố trên các tạp chí uy tín, bời vì các tổng biên tập không thích xuất bản những bài như thế. Ngược lại, một nghiên cứu với một kết quả "tích cực" thì nghiên cứu có khả năng xuất hiện trên các tạp chí khoa học cao hơn là các nghiên cứu với kết quả "tiêu cực". Phụ lục 4, trình bày kết quả của biểu đồ funnel và kiểm định Egger, cho thấy nếu trong trường hợp không có xu hướng thiên lệch trong xuất bản và các hiệu ứng nghiên cứu nhỏ, thì biểu đồ các nghiên cứu phải giống một hình phễu đảo ngược đối xứng, biểu đồ cho thấy có một vài nghiên cứu bị thiếu ở phần dưới bên trái của biểu đồ, điều này khiến nó trông không đối xứng, kết quả kiểm định Egger $\operatorname{Pr}(z)=0,000<0,05$, cho thấy có sự thiên vị trong xuất bản. Để làm rõ hơn vấn đề này, trong 30 nghiên cứu được chia thành 2 nhóm, Nhóm 0 là những nghiên cứu được đăng trên các tạp chí, hội thảo không thuộc danh mục ISI/SCOPUS. Nhóm 1 là những nghiên cứu được xuất bản trên các tạp chí quốc tế uy tín. Phụ lục 4, 5, 6 trình bày kết quả tổng hợp khi được phân nhóm dựa trên chỉ số xuất bản, kết quả cho thấy các nghiên cứu thuộc nhóm 0 , có hệ số tương quan cao hơn với $r=0,35$ cao hơn nhóm 1 với $r=0,27$, đồng thời hệ số $I^{2}=92,09 \%$ của nhóm 0 , cho thấy sự thiên lệch trong xuất bản cũng cao hơn.

\section{KẾT LUÂN}

Sử dụng phương pháp phân tích tổng hợp, nghiên cứu này xem xét mối quan hệ giữa SR và CFP. Tổng hợp kết quả nghiên cứu của 30 nghiên cứu thực nghiệm về mối liên kết giữa $S R$ và $C F P$, nghiên cứu này tán thành lập luận phổ biến rằng SR nâng cao CFP. Hơn nữa, các tác giả đã khám phá hướng của nguyên nhân. Các tác giả nhận thấy rằng kết quả hoạt động tài chính tiếp theo có liên quan tích cực với SR trước đó, hỗ trợ cho lý thuyết các bên liên quan. Các kết quả phân tích tổng thể cho thấy rằng hoạt động bền vững của doanh nghiệp có tác động tích cực đến hoạt động tài chính của doanh nghiệp theo cả hai chiều, đặc biệt là trong dài hạn. Điều này ngụ ý rằng về lâu dài, các công ty quan tâm đến SR sẽ có hiệu quả tài chính cao. Do đó có thể thúc đẩy các nhà quản lý theo đuổi SR mặc dù công ty có thể không có lãi trong ngắn hạn.

Các nghiên cứu SR đã được cải thiện theo thời gian với các lý thuyết lý thuyết mạnh mẽ hơn, các hoạt động phù hợp hơn và các biện pháp kiểm soát ngày càng tốt hơn đối với các biến bị bỏ qua trước đó. Đánh giá phân tích tổng hợp, cùng với các cuộc thảo luận sâu rộng và đề xuất nghiên cứu trong tương lai, có thể cung cấp tài liệu tham khảo để phát triển lý thuyết, thiết kế nghiên cứu và phân tích thực nghiệm tại hiện trường. Các tác giả hy vọng rằng đánh giá này làm rõ và củng cố kiến thức học thuật về $S R$ và giá trị của nó trong việc nâng cao hiệu quả hoạt động tài chính.

Nghiên cứu này có thể được mở rộng sang các lĩnh vực khác liên quan đến tính bền vững. Ví dụ, công bố thông tin về tính bền vững và quản trị công ty có thể được kiểm tra để điều tra tác động của chúng đối với kết quả hoạt động bền vững.

Tóm lại, nghiên cứu này đóng góp vào tài liệu về tính bền vững của doanh nghiệp hiện tại bằng cách cung cấp một cuộc điều tra chuyên sâu về các biện pháp khác nhau được sử dụng cho SR và CFP, kiểm tra chặt chẽ các đặc điểm của mẫu và so sánh các phương pháp khác nhau được sử dụng trong các tài liệu trước đây bằng cách sử dụng phân tích tổng hợp.

\section{PHU LUC}

Phụ lục 1. Các bài báo/báo cáo được sử dụng trong mẫu nghiên cứu mối quan hệ báo cáo bền vững và hiệu quả tải chính

\begin{tabular}{|l|l|l|l|l|}
\hline STT & Các tác giả & \multicolumn{1}{|c|}{ Tên bài báo } & $\begin{array}{l}\text { Năm } \\
\text { xuất } \\
\text { bản }\end{array}$ & Tạp chí \\
\hline 1 & $\begin{array}{l}\text { Arayssi M., } \\
\text { Dah M., Jizi } \\
\text { M. }\end{array}$ & $\begin{array}{l}\text { Women on boards, } \\
\text { sustainability reporting and } \\
\text { firm performance }\end{array}$ & 2016 & $\begin{array}{l}\text { Sustainability } \\
\text { Accounting, } \\
\text { Management and } \\
\text { Policy Journal }\end{array}$ \\
\hline 2 & $\begin{array}{l}\text { Dada E. T., } \\
\text { Onyeogaziri U. } \\
\text { R. }\end{array}$ & $\begin{array}{l}\text { Reporting on Corporate } \\
\text { Performance of Selected } \\
\text { Quoted Brewery Firms in } \\
\text { Nigeria }\end{array}$ & 2018 & $\begin{array}{l}\text { International } \\
\text { Journal of } \\
\text { Business \& Law } \\
\text { Research }\end{array}$ \\
\hline 3 & $\begin{array}{ll}\text { The Effect of Sustainability } \\
\text { Is sustainability reporting } \\
\text { (ESG) associated with } \\
\text { performance? Evidence from } \\
\text { the European banking sector }\end{array}$ & 2018 & $\begin{array}{l}\text { Management of } \\
\text { Environmental } \\
\text { Quality: An } \\
\text { International } \\
\text { Journal }\end{array}$ \\
\hline
\end{tabular}




\begin{tabular}{|c|c|c|c|c|}
\hline 4 & $\begin{array}{l}\text { Buallay A., } \\
\text { Fadel S. M., } \\
\text { Alajmi J., } \\
\text { Saudagaran S. }\end{array}$ & $\begin{array}{l}\text { Sustainability reporting and } \\
\text { bank performance after } \\
\text { financial crisis Evidence from } \\
\text { developed and developing } \\
\text { countries }\end{array}$ & 2020 & $\begin{array}{l}\text { Competitiveness } \\
\text { Review: An } \\
\text { International } \\
\text { Business Journal }\end{array}$ \\
\hline 5 & $\begin{array}{l}\text { Buallay A., } \\
\text { Hamdan A., } \\
\text { Barone E. }\end{array}$ & $\begin{array}{l}\text { Sustainability reporting and } \\
\text { firm's performance } \\
\text { Comparative study between } \\
\text { manufacturing and banking } \\
\text { sectors }\end{array}$ & 2019 & $\begin{array}{l}\text { International } \\
\text { Journal of } \\
\text { Productivity and } \\
\text { Performance } \\
\text { Management }\end{array}$ \\
\hline 6 & $\begin{array}{l}\text { Burhan A. H. } \\
\text { N., Rahmanti } \\
\text { W. }\end{array}$ & $\begin{array}{l}\text { The impact of sustainability } \\
\text { reporting on company } \\
\text { performance }\end{array}$ & 2012 & $\begin{array}{l}\text { Journal of } \\
\text { Economics, } \\
\text { Business, and } \\
\text { Accountancy } \\
\text { Ventura }\end{array}$ \\
\hline 7 & $\begin{array}{l}\text { Carp M., } \\
\text { Păvăloaia L., } \\
\text { Afrăsinei M. } \\
\text { B., Georgescu } \\
\text { I. E. } \\
\end{array}$ & $\begin{array}{l}\text { Is Sustainability Reporting a } \\
\text { Business Strategy for Firm's } \\
\text { Growth? Empirical Study on } \\
\text { the Romanian Capital } \\
\text { Market }\end{array}$ & 2019 & Sustainability \\
\hline 8 & $\begin{array}{l}\text { Ekwueme J. } \\
\text { A., Onuora J. } \\
\text { K. P. }\end{array}$ & $\begin{array}{l}\text { Sustainability accounting and } \\
\text { stock performance of quoted } \\
\text { consumer goods } \\
\text { manufacturing firms }\end{array}$ & 2019 & $\begin{array}{l}\text { Journal of Global } \\
\text { Accounting }\end{array}$ \\
\hline 9 & $\begin{array}{l}\text { Emeka- } \\
\text { Nwokeji N., } \\
\text { Osisioma B. C. }\end{array}$ & $\begin{array}{l}\text { Sustainability disclosures } \\
\text { and market value of firms in } \\
\text { emerging economy: } \\
\text { evidence from Nigeria }\end{array}$ & 2019 & $\begin{array}{l}\text { International } \\
\text { Journal of } \\
\text { Accounting, } \\
\text { Finance and Risk } \\
\text { Management }\end{array}$ \\
\hline 10 & Laskar N. & $\begin{array}{l}\text { Impact of Corporate } \\
\text { Sustainability Reporting on } \\
\text { Firm Performance: An } \\
\text { Empirical Examination in } \\
\text { Asia }\end{array}$ & 2018 & $\begin{array}{l}\text { Journal of Asia } \\
\text { Business Studies }\end{array}$ \\
\hline 11 & Najul Laskar & $\begin{array}{l}\text { Does Sustainability } \\
\text { Reporting Enhance Firms } \\
\text { Profitability? A Study on } \\
\text { Select Companies from India } \\
\text { and South Korea }\end{array}$ & 2019 & $\begin{array}{l}\text { Indian Journal of } \\
\text { Corporate } \\
\text { Governance }\end{array}$ \\
\hline 12 & Ngatia C. N. & $\begin{array}{l}\text { Exploring sustainability } \\
\text { reporting for financial } \\
\text { performance of selected } \\
\text { companies listed at the } \\
\text { nairobi securities exchange } \\
\text { in Kenya }\end{array}$ & 2014 & $\begin{array}{l}\text { International } \\
\text { Academic Journal } \\
\text { of Economics and } \\
\text { Finance }\end{array}$ \\
\hline 13 & $\begin{array}{l}\text { Uwuigbe U., } \\
\text { Teddy 0., } \\
\text { Uwuigbe 0. R., } \\
\text { Emmanuel 0., } \\
\text { Asiriuwa 0., } \\
\text { Eyitomi G. A., } \\
\text { Taiwo 0.S. }\end{array}$ & $\begin{array}{l}\text { Sustainability reporting and } \\
\text { firm performance: a bi- } \\
\text { directional approach }\end{array}$ & 2018 & $\begin{array}{l}\text { Academy of } \\
\text { Strategic } \\
\text { Management } \\
\text { Journal }\end{array}$ \\
\hline
\end{tabular}

\begin{tabular}{|c|c|c|c|c|}
\hline 14 & $\begin{array}{l}\text { Zahid M., } \\
\text { Rahman H. U., } \\
\text { Khan M., Ali } \\
\text { W., Shad F. }\end{array}$ & $\begin{array}{l}\text { Addressing endogeneity by } \\
\text { proposing novel } \\
\text { instrumental variables in } \\
\text { the nexus of sustainability } \\
\text { reporting and firm financial } \\
\text { performance: A step-by-step } \\
\text { procedure for non experts }\end{array}$ & 2020 & $\begin{array}{l}\text { Business Strategy } \\
\text { and the } \\
\text { Environment }\end{array}$ \\
\hline 15 & $\begin{array}{l}\text { Brammer S., } \\
\text { Brooks C., } \\
\text { Pavelin S. }\end{array}$ & $\begin{array}{lr}\text { Corporater Social } \\
\text { Performance and Stock } \\
\text { Returns: UK Evidence from } \\
\text { Disaggregate Measures } \\
\end{array}$ & 2006 & $\begin{array}{l}\text { Financial } \\
\text { Management }\end{array}$ \\
\hline 16 & $\begin{array}{l}\text { Dhaliwal D. S., } \\
\text { Li O. Z., Tsang } \\
\text { A., Yang Y. G. }\end{array}$ & $\begin{array}{l}\text { Voluntary Nonfinancial } \\
\text { Disclosure and the Cost of } \\
\text { Equity Capital: The Initiation } \\
\text { of Corporate Social } \\
\text { Responsibility Reporting }\end{array}$ & 2011 & $\begin{array}{l}\text { The accounting } \\
\text { review }\end{array}$ \\
\hline 17 & $\begin{array}{l}\text { Vijfvinkel S., } \\
\text { Bouman N., } \\
\text { Hessels J. }\end{array}$ & $\begin{array}{l}\text { Environmental sustainability } \\
\text { and financial performance of } \\
\text { SMEs }\end{array}$ & 2011 & $\begin{array}{l}\text { Scientific Analysis } \\
\text { of } \\
\text { Entrepreneurship } \\
\text { and SMEs }\end{array}$ \\
\hline 18 & $\begin{array}{l}\text { Bayoud N. S., } \\
\text { Kavanagh M., } \\
\text { Slaughter G. }\end{array}$ & $\begin{array}{l}\text { Corporate Social Responsibility } \\
\text { Disclosure and Corporate } \\
\text { Reputation in Developing } \\
\text { Countries: The Case of Libya }\end{array}$ & 2012 & $\begin{array}{l}\text { Journal of } \\
\text { Business and } \\
\text { Policy Research }\end{array}$ \\
\hline 19 & $\begin{array}{l}\text { Eccles R. G., } \\
\text { Ioannou I., } \\
\text { Serafeim G. }\end{array}$ & $\begin{array}{lr}\text { The impact of } & \text { corporate } \\
\text { sustainability } & \text { on } \\
\text { organizational } & \text { processes } \\
\text { and performance } & \\
\end{array}$ & 2012 & $\begin{array}{l}\text { Working paper } \\
\text { series }\end{array}$ \\
\hline 20 & $\begin{array}{l}\text { Rajput N., } \\
\text { Batra G., } \\
\text { Pathak R. }\end{array}$ & $\begin{array}{l}\text { Linking CSR and financial } \\
\text { performance: an empirical } \\
\text { validation }\end{array}$ & 2012 & $\begin{array}{l}\text { Problems and } \\
\text { Perspectives in } \\
\text { Management } \\
\end{array}$ \\
\hline 21 & Suttipun M. & $\begin{array}{l}\text { Triple Bottom Line Reporting } \\
\text { in Annual Reports: A Case } \\
\text { Study of Companies Listed on } \\
\text { the Stock Exchange of } \\
\text { Thailand (SET) }\end{array}$ & 2012 & $\begin{array}{l}\text { Asian Journal of } \\
\text { Finance \& } \\
\text { Accounting }\end{array}$ \\
\hline 22 & Aggarwal P. & $\begin{array}{l}\text { Impact of Sustainability } \\
\text { Performance of Company on } \\
\text { its Financial Performance: A } \\
\text { Study of Listed Indian } \\
\text { Companies }\end{array}$ & 2013 & $\begin{array}{l}\text { Global Journal of } \\
\text { Management and } \\
\text { Business } \\
\text { Research Finance }\end{array}$ \\
\hline 23 & Ghosh A. & $\begin{array}{l}\text { Corporate Sustainability and } \\
\text { Corporate } \\
\text { Performance: The Indian } \\
\text { Context }\end{array}$ & 2013 & Working Paper \\
\hline 24 & $\begin{array}{l}\text { Karlsson J., } \\
\text { Bäckström S. } \\
\text { L. }\end{array}$ & $\begin{array}{l}\text { Corporate Sustainability and } \\
\text { Financial Performance - The } \\
\text { influence of board diversity } \\
\text { in a Swedish context } \\
\end{array}$ & 2015 & Thesis \\
\hline 25 & $\begin{array}{l}\text { San Ong T., } \\
\text { Teh B. H., Ang } \\
\text { Y. W. }\end{array}$ & $\begin{array}{l}\text { The Impact of } \\
\text { Environmental } \\
\text { Improvements on the } \\
\text { Financial Performance of } \\
\text { Leading Companies Listed in } \\
\text { Bursa Malaysia }\end{array}$ & 2014 & $\begin{array}{l}\text { International } \\
\text { Journal of Trade, } \\
\text { Economics and } \\
\text { Finance }\end{array}$ \\
\hline
\end{tabular}




\begin{tabular}{|c|c|c|c|c|}
\hline 26 & Hussain N. & $\begin{array}{l}\text { Impact of sustainability } \\
\text { performance on financial } \\
\text { performance: an empirical } \\
\text { study of global fortune } \\
\text { (N100) firms }\end{array}$ & 2015 & Working Paper \\
\hline 27 & $\begin{array}{l}\text { Goel P., Misra } \\
\text { R. }\end{array}$ & $\begin{array}{l}\text { Sustainability Reporting in } \\
\text { India: Exploring Sectoral } \\
\text { Differences and Linkages } \\
\text { with Financial Performance }\end{array}$ & 2017 & $\begin{array}{l}\text { Vision: The } \\
\text { Journal of } \\
\text { Business } \\
\text { Perspective }\end{array}$ \\
\hline 28 & $\begin{array}{l}\text { Hoang Thi Viet } \\
\text { Ha, Vu Thi } \\
\text { Thuy Van, } \\
\text { Dang Ngoc } \\
\text { Hung } \\
\end{array}$ & $\begin{array}{l}\text { Impact of Social } \\
\text { Reponsibility Information } \\
\text { Disclosure on the Financial } \\
\text { Performance of Enterprises } \\
\text { in Vietnam }\end{array}$ & 2019 & $\begin{array}{l}\text { India journal of } \\
\text { finance }\end{array}$ \\
\hline 29 & $\begin{array}{l}\text { Van Linh N., } \\
\text { Hung D. N., } \\
\text { Dang T. B., } \\
\text { Van V. T. T., } \\
\text { Anh N. T. M. } \\
\end{array}$ & $\begin{array}{l}\text { The effects of business } \\
\text { efficiency to disclose } \\
\text { information of sustainable } \\
\text { development }\end{array}$ & 2019 & $\begin{array}{l}\text { Asian Economic } \\
\text { and Financial } \\
\text { Review }\end{array}$ \\
\hline 30 & Buallay A. M. & $\begin{array}{l}\text { Sustainability reporting and } \\
\text { bank's performance: } \\
\text { comparison between } \\
\text { developed and developing } \\
\text { countries }\end{array}$ & 2020 & $\begin{array}{l}\text { World Review of } \\
\text { Entrepreneurship, } \\
\text { Management and } \\
\text { Sustainable } \\
\text { Development }\end{array}$ \\
\hline
\end{tabular}

Phụ lục 2. Biểu đồ forest plot phản ánh mối quan hệ báo cáo bền vững và hiệu quả tài chính tổng thể

\begin{tabular}{|c|c|c|c|}
\hline Study & & $\begin{array}{l}\text { Effect Size } \\
\text { with 95\% Cl }\end{array}$ & $\begin{array}{l}\text { Weight } \\
(\%)\end{array}$ \\
\hline Arayssi, M., Dah, M. \& J Jizi, M. (2016). & = & $0.13[0.02,0.23]$ & 4.39 \\
\hline Asuquo, A. I, Dada, E. T., \& Onyeogazziri, U. R. (2018). & $\square$ & $0.39[-0.18,0.95]$ & 1.28 \\
\hline Buallay, A (2018). & - & $0.48[0.35,0.61]$ & 4.21 \\
\hline Buallay, A, Fadel, S. M., Alajmi, J., \& Saudagaran, S. (2020). & [ & $0.24[0.18,0.31]$ & 4.64 \\
\hline Bualla, A., Hamdan, A, \& Barone, E. (2019). & 【 & $0.13[0.07,0.20]$ & 4.64 \\
\hline Burhan, A. H. N. \& Rahmanti, W. (2012). & $\rightarrow-$ & $0.57[0.200 .93]$ & 2.25 \\
\hline Carp, M, Păvắlaaia, L., Afräsinei, M. B, \& Georgescu, I. E. (2019) & - & $0.04[-0.22,0.30]$ & 3.03 \\
\hline Ekxweme, J. A, \& Onuora, J. K. P. (2019). & $\longrightarrow$ & $0.68[0.23,1.13]$ & 1.76 \\
\hline Emeka-Nwokeji, N, \& Osisioma, B.C. (2019) & $\longrightarrow$ & $-1.67[1.10,223]$ & 1.28 \\
\hline Laskar, N. (2018). & - & $0.49[0.30,0.67]$ & 3.68 \\
\hline Najul Laskar (2019) & $\rightarrow$ & $0.34[0.07,0.62]$ & 2.92 \\
\hline Ngatia, C. . . (2014). & 口 & $0.06[0.01,0.12]$ & 4.67 \\
\hline Umighe, U, Teddy, O. Unvigbe, O. R, Emmanue, O, Asiruwa, O, Eyitomi, G. A, \& Taimo, O. S. (2018). & $\longrightarrow$ & $0.96[0.22,1.70]$ & 0.84 \\
\hline Zahid, M. Rahman, H U., Khan, M., Ai, W, \& Shad, F. (2020). & $\longrightarrow$ & $0.91[0.17,1.65]$ & 0.84 \\
\hline Brammer, S, Brooks, C, \& Pavelin, S. (2006). & - & $0.41[0.14,0.68]$ & 2.96 \\
\hline Dhaliwal, D. S. Li, O.Z, Tsang, A. \& Y Yang, Y. G. (2011). & घ & $0.01[-0.05,0.07]$ & 4.68 \\
\hline Vijivinkel, S, Bouman, N, \& Hessels, J. (2011). & = & $0.12[0.01,0.23]$ & 4.38 \\
\hline Bayoud, N. S, Kavanagh, M. \& Slaughter, G. (2012). & $\rightarrow-$ & $0.07[-0.25,0.39]$ & 2.54 \\
\hline Ecoles, R. G, loannou, I. \& Serafém, G. (2012). & - & $0.59[0.38,0.80]$ & 3.49 \\
\hline Rajput, N, Bata, G, \& Pathak, R. (2012). & - & $0.39[0.26,0.51]$ & 4.24 \\
\hline Suttipun, M. (2012). & - & $0.21[-0.08,0.50]$ & 2.83 \\
\hline Agganwal, P. (2013). & $\rightarrow-$ & $0.25[-0.07,0.57]$ & 2.54 \\
\hline Ghosh, A (2013). & - & $0.15[0.02,0.29]$ & 4.13 \\
\hline Karlsson, J., \& Băckstrởm, S. L. (2015). & - & $0.17[0.05,0.29]$ & 4.25 \\
\hline San Ong, T., Teh, B. H., \& Ang, Y. W. (2014). & $\square$ & $0.34[0.11,0.56]$ & 3.34 \\
\hline Hussain, N. (2015). & + & $0.45[0.25,0.65]$ & 3.59 \\
\hline Goel, P.\& \& Misa, R. (2017). & 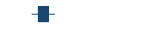 & $0.26[0.07,0.44]$ & 3.75 \\
\hline Hoang Thi Veet Ha, Vu Thi Thuy Van, Dang Nooc Hung (2019) & - & $0.17[0.05,0.28]$ & 4.31 \\
\hline Van Linh, N. Hung, D. . ., Dang, T. B., Van, V. T. T., \& An, N. T. M. (2019) & = & $0.23[0.11,0.34]$ & 4.32 \\
\hline Buallay, A. M. (2020). & - & $0.18[0.05,0.31]$ & 4.20 \\
\hline Overall & - & $0.29[0.21,0.36]$ & \\
\hline \multicolumn{4}{|l|}{ Heterogeneily. $I^{2}=0.03, I^{2}=87.53 \%, H^{2}=8.02$} \\
\hline \multicolumn{4}{|l|}{ Test of $\theta=\theta ; \alpha(29)=173.73, p=0.00$} \\
\hline \multicolumn{4}{|l|}{ Test of $\theta=0: z=7.50, p=0.00$} \\
\hline & 1 & & \\
\hline
\end{tabular}

Nguồn: Tính toán và chạy kết quả từ Stata 16
Phụ lục 3. Biểu đồ forest plot phản ánh mối quan hệ báo cáo bền vững và hiệu quả tài chính chia theo chiều hướng (0-Mỗi quan hễ giữa CFP - SR; 1- Mối quan hệ SR-CFP)

\begin{tabular}{|c|c|c|}
\hline Study & & $\begin{array}{l}\text { Effect Size } \\
\text { with 95\% Cl }\end{array}$ \\
\hline \multicolumn{3}{|l|}{0} \\
\hline Arayssi, M., Dah, M., \& J Jizi, M. (2016). & - & $0.13[0.02,0.23]$ \\
\hline Dhaliwa, D. S., Li, O.Z, Tsang, A, \& Yang, Y. G. (2011). & Ш & $0.01[-0.05,0.07]$ \\
\hline Sutipun, M. (2012). & - & $0.21[-0.08,0.50]$ \\
\hline Van Linh, N., Hung, D. N., Dang, T. B., Van, V. T. T., \& Anh, N. T. M. (2019) & - & $0.23[0.11,0.34]$ \\
\hline Heterogeneity. $I^{2}=0.01, I^{2}=74.32 \%, H^{2}=3.89$ & $\bullet$ & $0.12[0.01,0.23]$ \\
\hline
\end{tabular}

Testof $\theta=\theta ; Q(3)=13.25, p=0.00$

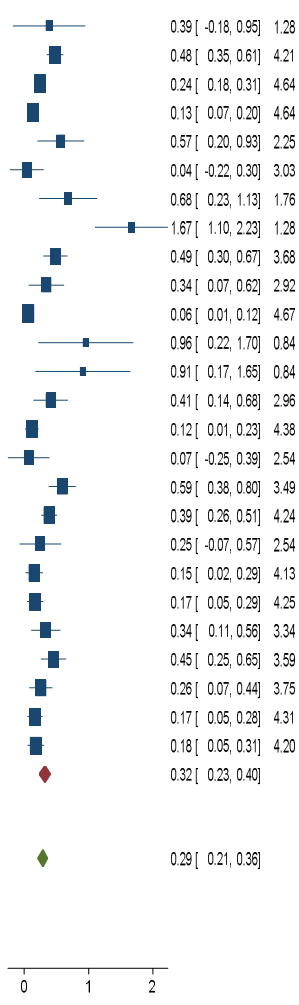

Nguồn: Tính toán và chạy kết quả từ Stata 16

Phụ lục 4. Biểu đồ Funnel plot và kiểm định Egger phản ánh mối quan hệ báo cáo bền vững và hiệu quả tài chính

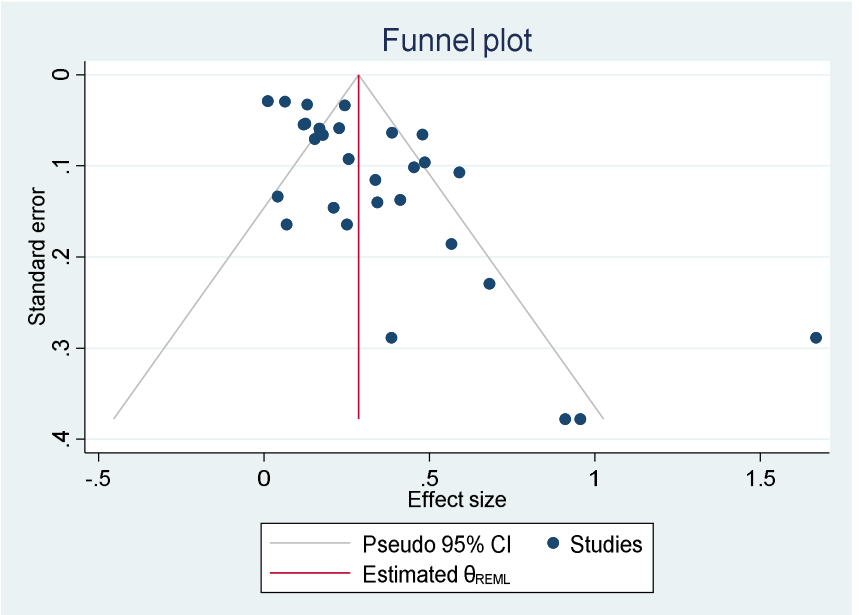




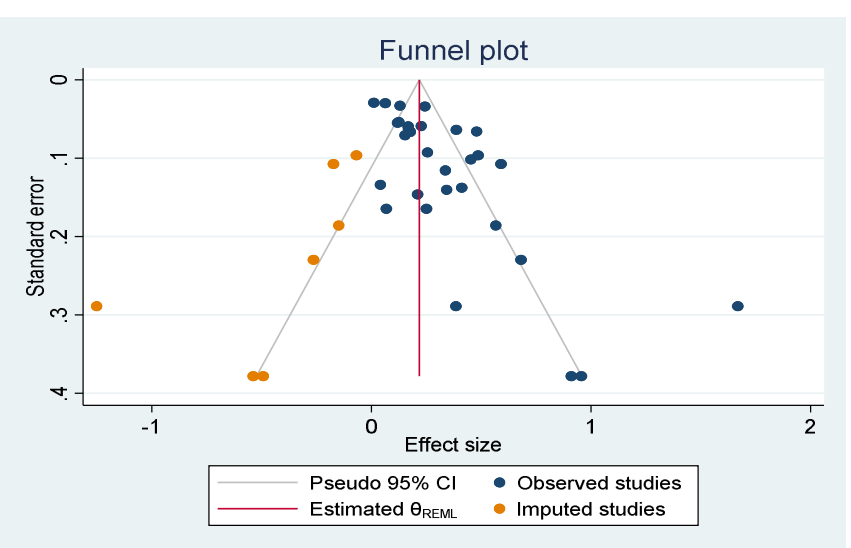

Regression-based Egger test for small-study effects Random-effects model

Method: REML

$$
\begin{array}{rlrl}
\text { HO: beta1 }=0 ; \text { no } & \text { small-study effects } \\
\text { beta1 } & = & 2.39 \\
\text { SE of beta1 } & = & 0.476 \\
z & = & 5.03 \\
\text { Prob }>|z| & = & 0.0000
\end{array}
$$

\begin{tabular}{|c|c|c|c|}
\hline Study & & $\begin{array}{l}\text { Effect Size } \\
\text { with } 95 \% \mathrm{Cl}\end{array}$ & $\begin{array}{l}\text { Weight } \\
(\%)\end{array}$ \\
\hline \multicolumn{4}{|l|}{0} \\
\hline Assuquo, A. I, Dada, E. T, \& Onyeogaziri, U. R. (2018). & $\longrightarrow$ & $0.39[-0.18,0.95]$ & 1.28 \\
\hline Burhan, A. H. . . \& Rahmant, W. (2012). & $\rightarrow$ & $0.57[0.20 .0 .93]$ & 2.25 \\
\hline Ekwweme, J. A, \& Onuora, J. K. . . (2019). & $\longrightarrow$ & $0.68[0.23,1.13]$ & 1.76 \\
\hline Emeka-Nwokej, N, \& Osisioma, B. C. (2019) & $\because$ & $-1.67[1.10,223]$ & 1.28 \\
\hline Ngatia, C. N. (2014). & च & $0.06[0.01,0.12]$ & 4.67 \\
\hline Vifininkel, S, Bouman, N. \& Hessels, J. (2011). & a & $0.12[0.010 .23]$ & 4.38 \\
\hline Bayoud, N. S., Kavanagh, M. \& Slaughter, G. (2012). & $\rightarrow$ & $0.07[-0.25,0.39]$ & 2.54 \\
\hline Eccles, R. G. loannou, L.\& \& Serafeim, G. (2012). & + & $0.59[0.38,0.80]$ & 3.49 \\
\hline Suttipun, M. (2012). & $\rightarrow$ & $0.21[-0.08,0.50]$ & 2.83 \\
\hline Aggarwal, P. (2013). & $\rightarrow-$ & $0.25[-0.07,0.57]$ & 2.54 \\
\hline Ghosh, A (2013). & = & $0.15[0.02,0.29]$ & 4.13 \\
\hline Karlsson, J. \& Bäckstrōom, S L. L. (2015). & 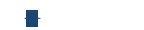 & $0.17[0.05,0.29]$ & 4.25 \\
\hline San Ong, T., Teh, B. H. \& Ang, Y. W. (2014). & - & $0.34[0.11,0.56]$ & 3.34 \\
\hline Heterogenenty. $I^{2}=0.09, I^{2}=9209 \%, H^{2}=1265$ & 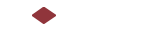 & $0.35[0.17,0.53]$ & \\
\hline \multicolumn{4}{|l|}{ Test of $\theta=\theta_{j} Q\{(12)=67.97, p=0.00$} \\
\hline \multicolumn{4}{|l|}{1} \\
\hline Arayssi, M. Dah, M. \& \& Jiz, M. (2016). & - & $0.13[0.02,0.23]$ & 4.39 \\
\hline Buallay, A. (2018). & $=$ & $0.48[0.35,0.61]$ & 4.21 \\
\hline Buallay, A, Fadel, S. M. A. Alajmi, J., \& Saudagaran, S. (2020). & ! & $0.24[0.18,0.31]$ & 4.64 \\
\hline Buallay, A, Hamdan, A, \& Barone, E (2019). & घ & $0.13[0.07,0.20]$ & 4.64 \\
\hline 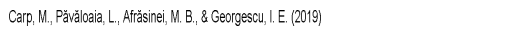 & - & $0.04[-0.22,0.30]$ & 3.03 \\
\hline Laskar, N. (2018). & - & $0.49[0.30,0.67]$ & 3.68 \\
\hline Najul Laskar (2019) & - & $0.34[0.07,0.62]$ & 2.92 \\
\hline 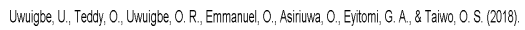 & $\longrightarrow$ & $0.96[0.22,1.70]$ & 0.84 \\
\hline Zanid, M, Rahman, H. U., Khan, M., Ali, W. \& \& Shad, F. (20202). & $\longrightarrow$ & $0.91[0.17,1.65]$ & 0.84 \\
\hline Brammer, S, Brooks, C, \& Pavelin, S. (2006). & $\rightarrow$ & $0.41[0.14,0.68]$ & 2.96 \\
\hline Dhaliwal, D. S, Li, O.Z, Tsang, A, \& Yang, Y. G. (2011). & 口 & $0.01[-0.05,0.07]$ & 4.68 \\
\hline Rajput, N. Batra, G. \& Pathak, R. (2012). & = & $0.39[0.26,0.51]$ & 4.24 \\
\hline Hussain, N. (2015). & - & $0.45[0.25,0.65]$ & 3.59 \\
\hline Goel, P. \& Missa, R. (2017). & $=$ & $0.26[0.07,0.44]$ & 3.75 \\
\hline Hoang Thi VietHa, Vu Thi Thuy Van, Dang Ngoc Hung (2019) & : & $0.17[0.05,0.28]$ & 4.31 \\
\hline Van Linh, N. Hung, D. N. Dang, T. B., Van, V. T. T., \& Anh, N. T. M. (2019) & - & $0.23[0.11,0.34]$ & 4.32 \\
\hline Buallay, A. M. (2020) & - & $0.18[0.05,0.31]$ & 4.20 \\
\hline Heterogeneity $I^{2}=0.02,1^{2}=84.64 \%, H^{2}=6.51$ & - & $0.27[0.19,0.35]$ & \\
\hline \multicolumn{4}{|l|}{ Test of $\theta=\theta_{j} ;(16)=103.89, p=0.00$} \\
\hline Overall & - & $0.29[0.21,0.36]$ & \\
\hline \multicolumn{4}{|l|}{$\begin{array}{l}\text { Heterogenenty }, I^{2}=0.03, I^{2}=87.53 \%, H^{2}=8.02 \\
\text { Test of } \theta=\theta ; Q 229=173.73, \mathrm{p}=0.00\end{array}$} \\
\hline Test of $\theta=\theta_{j} ;(29)=173.73, p=0.00$ & & & \\
\hline \multicolumn{4}{|l|}{ Test of group differences: $Q$ (. (1) $=0.68, p=0.41$} \\
\hline & 1 & & \\
\hline
\end{tabular}

Phụ lục 5. Biểu đồ forest plot phản ánh mối quan hệ báo cáo bền vững và hiệu quả tài chính theo chỉ số của tạp chí(1: Công bố trên tạp chí thuộc danh mục ISI/SCOPUS; 0: (ác công bố còn lại)

\begin{tabular}{|c|c|c|c|c|}
\hline Study & & $\begin{array}{l}\text { EtfectSize } \\
\text { with } 55 \% \mathrm{Cl}\end{array}$ & Prvalue & \\
\hline Asuquo, A. L., Dada, E. T., \& Onyeogazini, U. R. (2018). & $\cdot$ & $0.39[-0.18,0.95]$ & 0.182 & 0 \\
\hline Burhan, A. H. .N. \& \& Rahmant; W. (2012). & $\longrightarrow$ & $0.51[0.21,0.82]$ & 0.001 & 0 \\
\hline Ekwueme, J. A. \& \& Onuora, J. K. P. (2019). & $\rightarrow$ & $0.57[0.31,0.82]$ & 0.000 & 0 \\
\hline Emeka-Nwokej, W, \&Osisioma, B.C. (2019) & $\longrightarrow$ & $0.81[0.28,1.34]$ & 0.003 & 0 \\
\hline Ngatia, C. I. (2014). & $\longrightarrow$ & $0.64[0.12,1.15]$ & 0.015 & 0 \\
\hline Vifitinkel, S, Bouman, N, \&, \& Hessels, J. (2011). & $\longrightarrow$ & $0.54[0.10,0.98]$ & 0.017 & 0 \\
\hline Bayoud, N. S., Kavanagh, M. \& Slaughter, G. (2012). & $\longrightarrow$ & $0.47[0.08,0.86]$ & 0.019 & 0 \\
\hline Eccles, R. G., loannou, I., \& Serafeim, G. (2012). & $\longrightarrow$ & $0.48[0.14,0.81]$ & 10.005 & 0 \\
\hline Suttipun, 11. (2012). & $\longrightarrow$ & $0.44[0.15 ; 0.73]$ & 0.003 & 0 \\
\hline Agganwal, P. (2013). & $\longrightarrow$ & $0.42[0.16,0.67]$ & 0.001 & 0 \\
\hline Ghosh, A. (2013). & $\rightarrow$ & $0.38[0.15,0.61]$ & 0.001 & 0 \\
\hline Karlsson, J., \& Bäckström, S. L. (2015). & $\rightarrow$ & $0.36[0.15,0.56]$ & 0.001 & 0 \\
\hline San Ong, T., Teh, B. H. \& \& Ang, Y. W. (2014). & $\rightarrow$ & $0.35[0.17,0.53]$ & 0.000 & 0 \\
\hline Arayssi, M., Dah, M. \& J Jzi, M. (2016). & $\rightarrow$ & $0.32[0.16,0.49]$ & 0.000 & 1 \\
\hline Buallay, A. (2018). & $\rightarrow$ & $0.33[0.18,0.48]$ & 0.000 & 1 \\
\hline Buallay, A, Fadel, S. M, Alajmi, J, \& Saudagaran, S. (2020). & $\rightarrow$ & $0.32[0.19,0.45]$ & 0.000 & 1 \\
\hline Buallay, A, Hamdan, A, \& Barone, E, (2019). & $\rightarrow$ & $0.30[0.18,0.42]$ & 0.000 & 1 \\
\hline 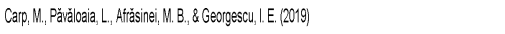 & $\rightarrow$ & $0.29[0.17,0.40]$ & 0.000 & 1 \\
\hline 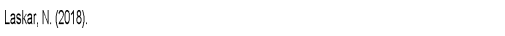 & $\rightarrow$ & $0.30[0.19,0.41]$ & 0.000 & 1 \\
\hline Najul Laskar (2019) & $\rightarrow$ & $0.30[0.19,0.40]$ & 0.000 & 1 \\
\hline 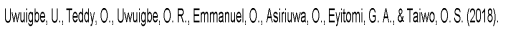 & $\rightarrow$ & $0.31[0.20,0.42]$ & 0.000 & 1 \\
\hline Zahici, M., Rahmen, H. U, Khan, M, Ali, W, \& Shad, F. (2020). & $\rightarrow$ & $0.32[0.21,0.43]$ & 0.000 & 1 \\
\hline Brammer,, , Brooks, C, \& Pavelin, S. (2006). & $\rightarrow$ & $0.32[0.22,0.43]$ & 0.000 & 1 \\
\hline Dhaliwa, , , S, L, L, , Z, , Tsang, A, \& Y Yang, Y, G, (2011). & $\rightarrow$ & $0.31[0.20,0.41]$ & 10.000 & 1 \\
\hline Rajput, N, Batra, G, \& Pathak, R. (2012). & $\rightarrow$ & $0.31[0.21,0.41]$ & 10.000 & 1 \\
\hline Hussain, N. (2015). & $\rightarrow$ & $0.31[0.22,0.41]$ & 0.000 & 1 \\
\hline Goel, P., \& Misia, R. (2017). & + & $0.31[0.22,0.40]$ & 0.000 & 1 \\
\hline Hoang Thi VietHa, Vu Thi Thuy Van, Dang Nooc Hung (2019) & + & $0.30[0.22,0.38]$ & 0.000 & 1 \\
\hline Van Linh, N, Hung, D. N., Dang, T. B, Van, V. . T. T., \& Anh, N. T. M. (2019) & + & $0.29[0.21,0.37]$ & 0.000 & 1 \\
\hline \multirow[t]{2}{*}{ Buallay, A. M. (2020). } & + & $0.29[0.21,0.36]$ & 0.000 & 1 \\
\hline & 0.5 & 1.5 & & \\
\hline
\end{tabular}

Nguồn: Tính toán và chạy kết quả từ Stata 16
Phụ lục 6 . Biểu đồ forest plot cumulative phản ánh mối quan hệ báo cáo bền vững và hiệu quả tài chính

Nguồn: Tính toán và chạy kết quả từ Stata 16

\section{TÀI LIỆU THAM KHẢO}

[1]. Albertini E., 2013. Does environmental management improve financial performance? A meta-analytical review. Organization \& Environment, 26(4), 431457.

[2]. Ameer R., Othman R., 2012. Sustainability practices and corporate financial performance: A study based on the top global corporations. Journal of Business Ethics, 108(1), 61-79.

[3]. Aras G., Aybars A., Kutlu 0., 2010. Managing corporate performance: Investigating the relationship between corporate social responsibility and financial performance in emerging markets. International Journal of productivity and Performance management, 59(3), 229-254.

[4]. Arayssi M., Dah M., Jizi M., 2016. Women on boards, sustainability reporting and firm performance. Sustainability Accounting, Management and Policy Journal, 7(3), 376-401. doi:https://doi.org/10.1108/SAMPJ-07-2015-0055

[5]. Asuquo A. I., Dada E., Onyeogaziri U., 2018. The effect of sustainability reporting on corporate performance of selected quoted brewery firms in Nigeria. International Journal of Business \& Law Research, 6(3), 1-10.

[6]. Aupperle K. E., Carroll A. B., Hatfield J. D., 1985. An empirical examination of the relationship between corporate social responsibility and profitability. Academy of management Journal, 28(2), 446-463. 
[7]. Buallay A., 2019. Is sustainability reporting (ESG) associated with performance? Evidence from the European banking sector. Management of Environmental Quality: An International Journal.

[8]. Burhan A. H. N., Rahmanti W., 2012. The impact of sustainability reporting on company performance. Journal of Economics, Business, and Accountancy Ventura, 15(2), 257-272.

[9]. Busse C., 2016. Doing well by doing good? The self-interest of buying firms and sustainable supply chain management. Journal of Supply Chain Management, 52(2), 28-47.

[10]. Carp M., Păvăloaia L., Afrăsinei M.-B., Georgescu I. E., 2019. Is sustainability reporting a business strategy for firm's growth? Empirical study on the Romanian capital market. Sustainability, 11(3), 658.

[11]. Chernev A., Blair S., 2015. Doing well by doing good: The benevolent halo of corporate social responsibility. Journal of Consumer Research, 41(6), 14121425.

[12]. Cho C. H., Freedman M., Patten D. M., 2012. Corporate disclosure of environmental capital expenditures. Accounting, auditing \& accountability journal. doi: https://doi.org/10.1108/09513571211209617

[13]. Cochran P. L., Wood R. A., 1984. Corporate social responsibility and financial performance. Academy of management Journal, 27(1), 42-56.

[14]. Cong Y., Freedman M., 2011. Corporate governance and environmental performance and disclosures. Advances in Accounting, 27(2), 223-232.

[15]. Crisóstomo V. L., de Souza Freire F., de Vasconcellos F. C., 2011. Corporate social responsibility, firm value and financial performance in Brazil. Social Responsibility Journal.

[16]. Davis K., 1973. The case for and against business assumption of social responsibilities. Academy of management Journal, 16(2), 312-322.

[17]. Deegan C., 2002. The legitimising effect of social and environmental disclosures-a theoretical foundation. Accounting, auditing \& accountability journal, 15(3), 282-311. doi:https://doi.org/10.1108/09513570210435852

[18]. Dixon-Fowler H. R., Slater D. J., Johnson J. L., Ellstrand A. E., Romi A. M., 2013. Beyond "does it pay to be green?" A meta-analysis of moderators of the CEP-CFP relationship. Journal of Business Ethics, 112(2), 353-366.

[19]. Ekwueme J. A., Onuora J. K. P., 2019. Sustainability accounting and stock performance of quoted consumer goods manufacturing firms. Journal of Global Accounting Department of Accountancy, 6(2).

[20]. Freeman R. E., 1984. Strategic Management: A Stakeholder Approach (Pitman, Boston, MA).

[21]. Friedman M., 1970. The social Responsibility of Business is to Increase its Profits. The New York Times Magazine, September 13, 173-178.

[22]. Galdeano-Gómez E., 2008. Does an endogenous relationship exist between environmental and economic performance? A resource-based view on the horticultural sector. Environmental and resource economics, 40(1), 73-89. doi:DOI 10.1007/s10640-007-9141-4

[23]. Haffar M., Searcy C., 2017. Classification of trade-offs encountered in the practice of corporate sustainability. Journal of Business Ethics, 140(3), 495-522.

[24]. Hahn R., Kühnen M., 2013. Determinants of sustainability reporting: a review of results, trends, theory, and opportunities in an expanding field of research. Journal of Cleaner Production, 59, 5-21.

[25]. He C., Loftus J., 2014. Does environmental reporting reflect environmental performance?: Evidence from China. Pacific Accounting Review 26(1-2), 134-154. doi:https://doi.org/10.1108/PAR-07-2013-0073
[26]. Hedges L. V., Vevea J. L., 1998. Fixed-and random-effects models in meta-analysis. Psychological methods, 3(4), 486.

[27]. Higgins J. P., Thompson S. G., 2002. Quantifying heterogeneity in a meta-analysis. Statistics in medicine, 21(11), 1539-1558.

[28]. Hoang T. V. H., Vu T. T. V., Dang, N. H., 2019. Impact of Social Reponsibility Information Disclosure on the Financial Performance of Enterprises in Vietnam. Indian Journal of Finance, 13(1), 20-36. doi:10.17010/ijf\%2F2019\%2Fv13i1\%2F141017

[29]. Holbrook M. E., 2010. Corporate social responsibility and financial performance: An examination of economic benefits and costs as manifested in accounting earnings. University of Kentucky.

[30]. Hunter J. E., Schmidt F. L., 2004. Methods of meta-analysis: Correcting error and bias in research findings. Sage.

[31]. Jaggi B., Freedman M., 1992. An examination of the impact of pollution performance on economic and market performance: pulp and paper firms. Journal of Business Finance \& Accounting, 19(5), 697-713.

[32]. Jensen M. C., Meckling W. H., 1976. Theory of the firm: Managerial behavior, agency costs and ownership structure. Journal of financial economics, $3(4), 305-360$.

[33]. Jones S., Frost G., Loftus J., van der Laan S., 2007. An empirical examination of the market returns and financial performance of entities engaged in sustainability reporting. Australian Accounting Review, 17(41), 78-87. doi:https://doi.org/10.1111/j.1835-2561.2007.tb00456.x

[34]. King A. A., Lenox M. J., 2001. Does it really pay to be green? An empirical study of firm environmental and financial performance: An empirical study of firm environmental and financial performance. Journal of Industrial Ecology, 5(1), 105116.

[35]. Lourenço I. C., Branco M. C., Curto J. D., Eugénio T., 2012. How does the market value corporate sustainability performance?. Journal of Business Ethics, 108(4), 417-428.

[36]. Lu W., Taylor M. E., 2016. Which factors moderate the relationship between sustainability performance and financial performance? A meta-analysis study. Journal of International Accounting Research, 15(1), 1-15.

[37]. McWilliams A., Siegel D., 2000. Corporate social responsibility and financial performance: correlation or misspecification? Strategic Management Journal, 21(5), 603-609.

[38]. Morhardt J. E., 2010. Corporate social responsibility and sustainability reporting on the internet. Business strategy and the environment, 19(7), 436-452.

[39]. Nelling E., Webb E., 2009. Corporate social responsibility and financial performance: the "virtuous circle" revisited. Review of Quantitative Finance and Accounting, 32(2), 197-209.

[40]. Orlitzky M., Schmidt F. L., Rynes, S. L., 2003. Corporate social and financial performance: A meta-analysis. Organization studies, 24(3), 403-441.

[41]. Preston L. E., O'bannon D. P., 1997. The corporate social-financial performance relationship: A typology and analysis. Business \& Society, 36(4), 419429.

[42]. Rhou Y., Singal M., Koh Y., 2016. CSR and financial performance: The role of CSR awareness in the restaurant industry. International Journal of Hospitality Management, 30-39. doi:https://doi.org/10.1016/j.ijhm.2016.05.007

[43]. Shank T. M., Shockey B., Financial R. J., 2016. Investment strategies when selecting sustainable firms. Financ. Serv. Rev, 25(2), 199-214. 
[44]. Simnett R., Vanstraelen A., Chua W. F., 2009. Assurance on sustainability reports: An international comparison. The Accounting Review, 84(3), 937-967.

[45]. Teoh S. H., Welch I., Wazzan C. P., 1999. The effect of socially activist investment policies on the financial markets: Evidence from the South African

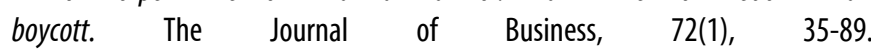
doi:https://www.jstor.org/stable/10.1086/209602

[46]. Ullmann A. A., 1985. Data in search of a theory: A critical examination of the relationships among social performance, social disclosure, and economic performance of US firms. Academy of Management review, 10(3), 540-557.

[47]. Van Linh N., Hung D. N., Dang T. B., Van V. T. T., Anh N. T. M., 2019. The Effects of Business Efficiency to Disclose Information of Sustainable Development: The Case of Vietnam. Asian Economic and Financial Review, 9(4), 547-558.

[48]. Waddock S. A., Graves S. B., 1997. The corporate social performancefinancial performance link. Strategic Management Journal, 18(4), 303-319.

[49]. Wang Q., Dou J., Jia S., 2016. A meta-analytic review of corporate social responsibility and corporate financial performance: The moderating effect of contextual factors. Business \& Society, 55(8), 1083-1121.

[50]. Wood D. J., 2010. Measuring corporate social performance: A review. International Journal of Management Reviews, 12(1), 50-84.

[51]. Wright P., Ferris S. P., 1997. Agency conflict and corporate strategy: The effect of divestment on corporate value. Strategic Management Journal, 18(1), 7783.

AUTHORS INFORMATION

Nguyen Van Linh, Dang Ngoc Hung, Dang Thi Hau

Hanoi University of Industry 